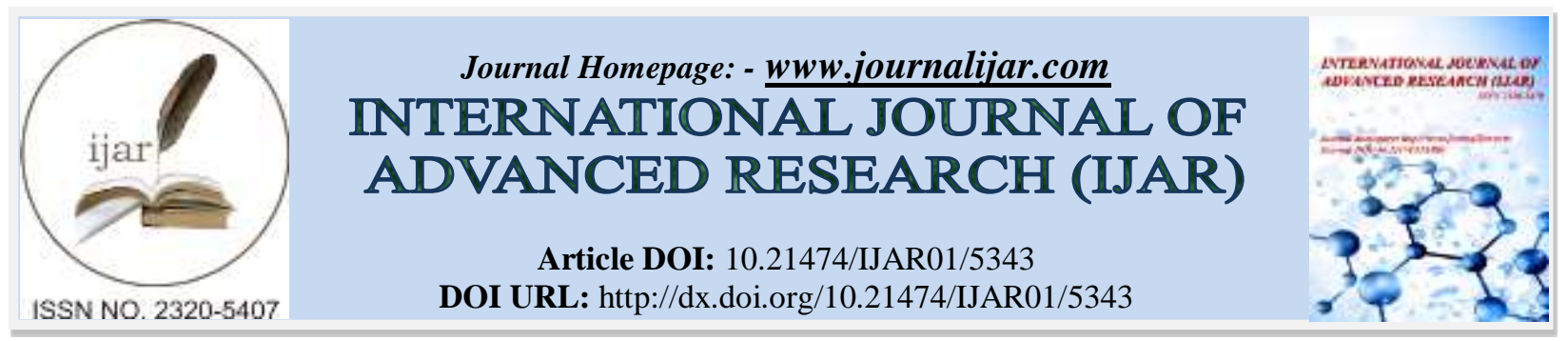

RESEARCH ARTICLE

\title{
REFRACTORY THROMOBOTIC ANTI-PHOSPHOLIPID SYNDROME MANIFESTING WITH BUDD CHIARI SYNDROME AND PULMONARY EMBOLISM.
}

\author{
Sherif A. Nasef, , Amer S. Zahralliyali, Mazen A. Elyahia, Ahmed Y. Alothman and Hanan M.Alrammah \\ Department of Medicine, King Fahad Specialist Hospital-Dammam, Saudi Arabia.
}

\section{Manuscript Info}

Manuscript History

Received: 06 July 2017

Final Accepted: 08 August 2017

Published: September 2017

Key words:-

Anti-phospholipid syndrome (APS), thrombosis, Pulmonary Embolism(PE), Inferior vena cava (IVC) thrombosis, Budd-Chiari Syndrome.

\section{Abstract}

Copy Right, IJAR, 2017,. All rights reserved.

\section{Introduction:-}

Anti-phospholipid syndrome (APS) is an auto-immune disease manifested by recurrent arterial or venous thrombosis and/or obstetric comorbidities. It affects approximately $0.5 \%$ of the general population [1]. Around 1-5\% of childbearing age women have detectable anti-phospholipid antibodies (aPL). Current treatment for APS is long-term anticoagulation in order to prevent further thrombotic events. A significant rate of APS patients develops recurrent events despite adequate treatment. The yearly incidence of recurrent events among APS patients receiving anticoagulation therapy ranges between 3 and 24\% in prospective studies and 52-69\% in retrospective studies [1]. We report a patient with refractory APS manifesting with Budd Chiari syndrome, which developed pulmonary embolism despite being on adequate warfarin therapy.

Case description:-

A 34-year-old Saudi female, presented to the emergency department with one-day history of severe epigastric pain, sharp in character, radiating to the back, associated with nausea and vomiting. For a month prior to that, she had progressive abdominal distention and lower limb edema without change of bowel habits, jaundice or melena/hematochezia. She was not on any medications and had no similar attacks previously. No significant past medical or surgical history. No alcohol, smoking or illicit drugs. Two of her brothers have bleeding disorder, and a maternal aunt died from complications of systemic lupus erythematosus (SLE).

In the emergency department, she was vitally stable and her examination was significant for distended abdomen with moderate epigastric tenderness but no rebound tenderness or rigidity. Her liver was felt $3 \mathrm{~cm}$ below the costal margin in right midclavicular line with palpable spleen tip. She had positive shifting dullness, and bilateral lower leg pitting edema. Laboratory tests showed normal CBC, liver panel, kidney functions and electrolytes with negative hepatitis serologies. 
Abdominal CT showed non-visualization of the intrahepatic veins; however the portal vein and hepatic arteries are well-opacified with no filling defect. There was a filling defect seen in the infra-diaphragmatic inferior vena cava (IVC) extending to the renal level with partial filling defect of the left renal vein. Another filling defect was seen below the renal level extending to the IVC bifurcation and the left common iliac vein. The liver was enlarged with hypertrophied caudate lobe and heterogeneous enhancement. There were multiple enhancing hepatic nodules in the arterial phase with low density central that appear hypodense in portovenous phase, the largest seen in the right hepatic lobe measuring $2.9 \times 1.6 \mathrm{~cm}$. The spleen was enlarged measuring $15 \mathrm{~cm}$ with no focal lesion. There was moderate to severe ascites.

Budd Chiari syndrome was diagnosed with extensive IVC thrombosis that partially involved the left renal vein and complete occlusion of the left common iliac vein. The hypervascular hepatic lesions represented regeneration nodules as a sequela of chronic Budd Chiari syndrome. Hypercoagulation work up showed positive lupus anticoagulants, beta-2 glycoprotein I IgG \& $\mathrm{M}$ and anticardiolipin IgG and IgM. Autoimmune work up showed positive ANA (1:640, speckled pattern) but negative anti ds-DNA Abs and normal complement levels.

She was diagnosed with primary antiphospholipid syndrome leading to Budd Chiari syndrome and she was sent to interventional radiology for thrombolysis. Cavogram was done through an infusion catheter which revealed filling defects in the IVC. Mechanical thrombolysis was done with inflation of multiple balloons to crush the thrombus then cavogram was repeated and showed significant short segment stenosis in the distal part of IVC which was treated with balloon angioplasty followed by repeated suction of the thrombus. Cavogram was then repeated and revealed good response. Multiple mechanical (balloon and suction) thrombolysis were done for the thrombosed segment of left common iliac vein as well as the proximal part of IVC, followed by venogram which showed partial response. Infusion catheter was placed through the thrombosed segment of left common iliac vein and connected to tPA infusion pump. Follow-up cavogram 8 hours later showed more than $50 \%$ recanalization of left common iliac vein. Maintenance dose of tPA through iliac infusion catheter. The patient was started on an Intravenous (IV) heparin drip after thrombolysis procedure with warfarin overlap. After 2 days of therapeutic INR heparin drip was stopped and the patient was discharged home on warfarin. The patient remained asymptomatic for 4 years on warfarin with therapeutic INR before she presented to the emergency department with coughing up bright red blood. She had no chest pain, shortness of breath, fever, night sweats, weight loss, or change in the appetite. Her vital signs were: Temperature 36.6 C, Pulse: $110 / \mathrm{min}$, BP: $131 / 83$, respiratory rate $26 / \mathrm{min}$ and oxygen saturation $95 \%$ on room air. Her physical examination was only significant for left basal crackles. Labs showed WBC: 7.6, HGB: 12.2, HCT: $37 \%$, MCV: 77, Platelet count: 255K and INR: 3.9. Chest X-ray and echocardiogram were normal. CT-angiogram of the chest (figures 1 and 2) showed evidence of at least two segmental pulmonary arterial filling defects involving the left upper and lower lobes branches associated with a perfusion abnormality. Such findings confirmed pulmonary embolism while on warfarin with supratherapeutic INR. The patient was admitted to general medical ward and hematology was consulted. She was started on SQ enoxaparin $1 \mathrm{mg} / \mathrm{kg} / 12 \mathrm{hr}$ and aspirin $81 \mathrm{mg}$ daily instead of warfarin. The patient improved clinically and hemoptysis resolved and she was discharged on SQ enoxaparin $1.5 \mathrm{mg} / \mathrm{kg} / \mathrm{d}$ and aspirin $81 \mathrm{mg}$ daily. The patient remained asymptomatic on this regimen for over two years now.

\section{Discussion:-}

The risk of thrombosis in APS depends on several factors. Anti-phospholipid-Anti-bodies (aPL) are one of the factors that influences the thrombotic risk in those patients ${ }^{[2]}$. Patients with triple-positive aPL-profile including lupus anticoagulant (LAC), anticardiolipin (aCL) antibodies, and anti $\beta 2$ glycoprotein I (a $\beta 2$ GPI) antibodies -as in our patient- have higher thrombotic risk compared to those with a single positive aPL-profile ${ }^{[1]}$. However, other factors such as co-existent auto-immune disease, hypertension, smoking, hypercholesterolemia and estrogen use have an impact on the risk of thrombosis ${ }^{[3]}$. The desired INR for these patients has been debated. An INR of $2.0-$ 3.0 has been suggested as a target in patients who present with venous thrombosis while an INR of $3.0-4.0$ is desired in those with arterial thrombosis [4]. However, there are no enough studies available to support this recommendation ${ }^{[5]}$. Moreover, such high INR is associated with increased risk of bleeding ${ }^{[4]}$. There is only scarce evidence on the treatment approach for patients with refractory APS (who have recurrent obstetric complications or recurrent thrombosis despite adequate anti-coagulation treatment) ${ }^{[4]}$, such as our case. Thus treatment depends largely on the clinical judgment. Previous studies have shown that many patients on warfarin who presented with recurrent thrombotic events had an INR less than 3.0 at the time of the event, and the rate of thrombotic events was lower in patients with an INR of 3.0 - 4.0. Nevertheless, studies by Crowther et al. and Finazzi et al. showed no difference in the outcome between patients on conventional anticoagulation (INR $2.0-3.0$ ) and high-intensity 
anticoagulation (INR > 3.0) ${ }^{[6]}$. According to the 14th International Congress on Antiphospholipid Antibodies task force report on antiphospholipid syndrome treatment trends, the first step in managing APS patients with recurrent thrombosis is to ensure that the target INR (2.0-3.0) is achieved, followed by increasing intensity of oral anticoagulation therapy to target INR > 3.0 and if this fails, switching oral anticoagulation to subcutaneous LMWH or adding aspirin, statin or hydroxychloroquine to the oral anti-coagulation can be attempted ${ }^{[5]}$. Rituximab has been used in some cases in order to decrease aPL titer, and this has shown variable efficacy ${ }^{[4]}$ Rituximab is a B-cell depleting biologic agent, thus suggesting a role of B-cells in APS pathogenesis ${ }^{[4]}$.

\section{Conclusion:-}

The treatment approach for patients with refractory APS who have recurrent obstetric complications or recurrent thrombosis despite adequate anti-coagulation treatment is challenging. Although recurrence of thrombosis yields an increased mortality, univocal recommendations to manage these situations are lacking. Intensifying oral anticoagulant therapy or the addition of low dose aspirin, hydroxychloroquine and statins to standard oral anticoagulation may lead to a better disease control. Moreover, long-term LMWH is also considered as a safe and effective alternative to standard oral anticoagulation. IVIG and B-cell based biologics are promising in the management of APS and aPL-related clinical manifestations. Research to identify novel therapeutic targets is ongoing as such pharmacological agents might revolutionize APS management in the near future.

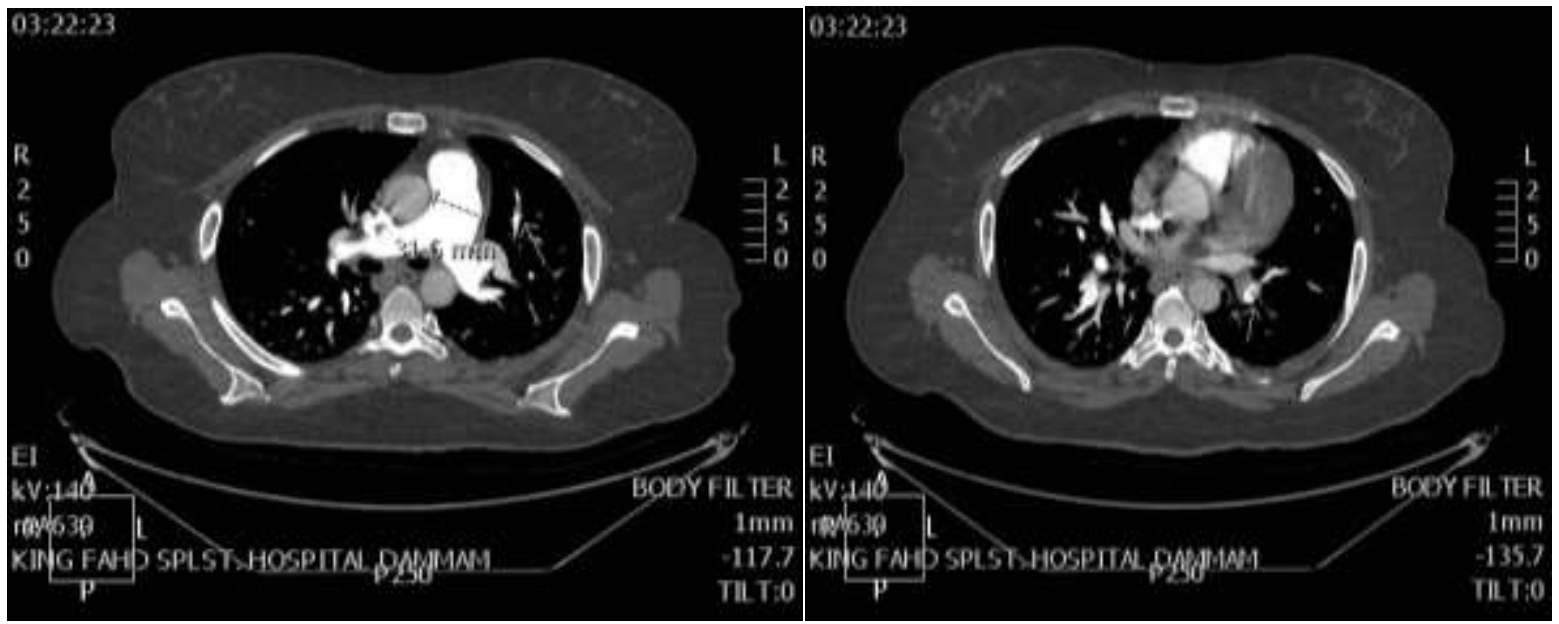

Figure 1 and 2:- Two filling defects in the left upper lobe and left lower lobe segmental pulmonary artery branches consistent with pulmonary embolism. (White arrows).

\section{References:-}

1. Chighizola C, Ubiali T, Meroni P. Treatment of Thrombotic Antiphospholipid Syndrome: The Rationale of Current Management-An Insight into Future Approaches. Journal of Immunology Research. 2015;2015:1-20. doi:10.1155/2015/951424.

2. MIYAKIS S, LOCKSHIN M, ATSUMI $T$ et al. International consensus statement on an update of the classification criteria for definite antiphospholipid syndrome (APS). Journal of Thrombosis and Haemostasis. 2006;4(2):295-306. doi:10.1111/j.1538-7836.2006.01753.x.

3. Roubey R. Risky business: the interpretation, use, and abuse of antiphospholipid antibody tests in clinical practice. Lupus. 2010;19(4):440-445. doi:10.1177/0961203310361356.

4. Scoble T, Wijetilleka S, Khamashta M. Management of refractory anti-phospholipid syndrome. Autoimmunity Reviews. 2011;10(11):669-673. doi:10.1016/j.autrev.2011.04.030.

5. Espinosa G, Cervera R. Current treatment of antiphospholipid syndrome: lights and shadows. Nature Reviews Rheumatology. 2015;11(10):586-596. doi:10.1038/nrrheum.2015.88.

6. Crowther M, Ginsberg J, Julian J. A comparison of two intensities of warfarin for the prevention of recurrent thrombosis in patients with the antiphospholipid antibody syndrome. ACC Current Journal Review. 2004;13(1):15. doi:10.1016/j.accreview.2003.10.079. 